4-1-1994

\title{
Phase Diagram of the Ising Model on Percolation Clusters
}

\author{
Miron Kaufman \\ Cleveland State University, m.kaufman@csuohio.edu \\ T. Berger \\ P. D. Gujrati \\ D. Bowman
}

Follow this and additional works at: https://engagedscholarship.csuohio.edu/sciphysics_facpub

Part of the Physics Commons

How does access to this work benefit you? Let us know!

Publisher's Statement

Copyright 1994 American Physical Society. Available on publisher's site at http://link.aps.org/ doi/10.1103/PhysRevB.49.9583.

\section{Original Citation}

Kaufman, Miron, T. Berger, P. D. Gujrati, and D. Bowman. "Phase Diagram of the Ising Model on Percolation Clusters." Physical Review B 49 (1994): 9583-9585.

\section{Repository Citation}

Kaufman, Miron; Berger, T.; Gujrati, P. D.; and Bowman, D., "Phase Diagram of the Ising Model on Percolation Clusters" (1994). Physics Faculty Publications. 5.

https://engagedscholarship.csuohio.edu/sciphysics_facpub/5

This Article is brought to you for free and open access by the Physics Department at EngagedScholarship@CSU. It has been accepted for inclusion in Physics Faculty Publications by an authorized administrator of

EngagedScholarship@CSU. For more information, please contact library.es@csuohio.edu. 


\title{
Phase diagram of the Ising model on percolation clusters
}

\author{
Miron Kaufman and Jimmy E. Touma* \\ Department of Physics, Cleveland State University, Cleveland, Ohio 44115
}

(Received 12 November 1993)

\begin{abstract}
The annealed Ising magnet on percolation clusters is studied by means of a mapping into a Potts-Ising model and with the Migdal-Kadanoff renormalization-group method. The phase diagram is determined in the three-dimensional parameter space of the Ising coupling $K$, the bond-occupation probability $p$, and the fugacity $q$, which controls the number of clusters. Three phases are identified: percolating ferromagnetic, percolating paramagnetic, and nonpercolating paramagnetic. For large $q$ the phase diagram includes a multicritical point at the intersection of the Ising critical line and the percolation critical line. In the case of random bond percolation $(q=1)$ the Ising critical line is: $p\left(1-e^{-2 K}\right)=1-\exp \left(-2 L_{C}\right)$, where $L_{C}$ is the pure-Ising-model critical coupling.
\end{abstract}

\section{INTRODUCTION}

The Ising model defined on percolation clusters is relevant to a variety of physical phenomena such as phase separation in a binary fluid mixture in a porous medium $^{1}$ and amorphous magnets. In this paper we analyze the annealed Ising model on percolation clusters; i.e., we assume that the relaxation times for spin configurations and for percolation configurations are short compared to the characteristic time of measurement. The percolation part of this model is a generalization $^{2}$ of the random-bond percolation which includes a fugacity $q$ that controls the number of clusters. This generalized percolation model is employed in Monte Carlo ${ }^{3}$ and renormalization-group ${ }^{4}$ studies of the Potts model. ${ }^{5}$

In Sec. II we define the model and present some exact results. In the particular case of the square lattice the Ising critical line for the Ising model on random-bond (fugacity $q=1)$ percolation clusters is $p\left(1-e^{-2 K}\right)$ $=2-\sqrt{2}$, where $K$ is the Ising coupling constant and $p$ is the bond-occupation probability.

In Sec. III we present a numerical analysis of the model in two-dimensions (2D) based on the Migdal-Kadanoff renormalization-group technique. ${ }^{6}$ Though only approximate for the 2D Bravais lattice this technique is exact ${ }^{7}$ for a hierarchical lattice. ${ }^{8}$ Hence the exact results presented in Sec. II are satisfied by this technique thus enhancing our confidence that the numerical findings are qualitatively correct. We present the phase diagram in the Ising coupling $K$, the bond-occupation probability $p$ plane for fixed fugacity $q$. For all $q$ we find three phases: the paramagnetic nonpercolating, the paramagnetic percolating, and the ferromagnetic percolating. If $q$ is small enough the Ising critical line and the percolation critical line intersect at zero temperature. If $q$ is sufficiently large the two lines meet at a multicritical point at finite temperature. In this case the ferromagnetic percolating phase can be accessed directly from the paramagnetic nonpercolating phase by crossing a line in the universality class of the $2 q$-state Potts model.

The summary of our findings as well as a critique are presented in Sec. IV.

\section{MODEL AND EXACT RESULTS}

At each site $i$ of a lattice there is an Ising spin $s_{i}= \pm 1$. The lattice sites are connected by bonds according to the generalized bond-percolation model: ${ }^{2}$ a bond is present with a probability $p$ and percolation configurations with the same number of bonds are distinguished according to the number of clusters $C$ as the configuration probability is proportional to $q^{C}$. For a given percolation configuration the partition function of the Ising model is

$$
Z_{I}^{\text {conf }}=\operatorname{Tr}_{\left\{s_{i}\right\}} \exp \left[K \sum_{\langle i, j\rangle}\left(s_{i} s_{j}-1\right)\right],
$$

where $\langle i, j\rangle$ denotes a pair of sites connected by a bond. The partition function for the annealed Ising magnet on percolation clusters is

$$
Z=\sum_{\text {conf. }} q^{C} w^{B} Z_{I}^{\text {conf }},
$$

where $B$ is the number of bonds, $C$ is the number of clusters including single-site clusters, and $w=p /(1-p)$.

By using the Kasteleyn-Fortuin ${ }^{9}$ expansion for the Potts model we map the model defined in Eqs. (1) and (2) into a Potts-Ising model. At each site $i$ there is the Potts spin $\sigma_{i}$ taking $q$ values and the Ising spin $s_{i}$ taking two values. The partition function is

$$
Z=\operatorname{Tr}_{\{s\}} \operatorname{Tr}_{\{\sigma\}} \prod_{\langle i, j\rangle}\left\{1+\delta\left(\sigma_{i}, \sigma_{j}\right) w \exp \left[K\left(s_{i} s_{j}-1\right)\right]\right\}
$$

where $\delta$ is the Kronecker delta and $\Pi$ is a product over all lattice edges. By writing the factor on the right-hand side of Eq. (3) as a Boltzmann factor

$$
\begin{aligned}
1+\delta\left(\sigma_{i}, \sigma_{j}\right) w \exp \left[K\left(s_{i} s_{j}-1\right)\right] \\
=\exp \left[J \delta\left(\sigma_{i}, \sigma_{j}\right)+L \delta\left(\sigma_{i}, \sigma_{j}\right) s_{i} s_{j}\right],
\end{aligned}
$$

we find

$$
\begin{aligned}
& J=\frac{1}{2} \ln \left[(1+w)\left(1+w e^{-2 K}\right)\right], \\
& L=\frac{1}{2} \ln \left[(1+w) /\left(1+w e^{-2 K}\right)\right] .
\end{aligned}
$$


The model is now given by the Hamiltonian

$$
-H / k_{B} T=\sum_{\langle i, j\rangle}\left[J \delta\left(\sigma_{i}, \sigma_{j}\right)+L \delta\left(\sigma_{i}, \sigma_{j}\right) s_{i} s_{j}\right] .
$$

Certain exact results can be obtained by studying the Ising-Potts Hamiltonian of Eq. (6). If $K=0$, i.e., infinite temperature, from Eq. (5) it follows that $L=0$, and the model reduces to the $q$-state Potts model. ${ }^{5}$ The Potts critical coupling for the square lattice is (Ref. 5) $J_{C}=\ln (1+\sqrt{q})$. Equation (5) then implies $w_{C}=\sqrt{q}$ or the threshold bond-occupation probability is $p_{C}=\sqrt{q} /(1+\sqrt{q})$. For the random-bond percolation $q=1$ and thus $p_{C}=\frac{1}{2}$.

If $K=\infty$, or zero temperature, Eqs. (5) imply $J=L$, and thus the Hamiltonian becomes

$$
-H / k_{B} T=\sum_{\langle i, j\rangle} 2 J \delta\left(\sigma_{i}, \sigma_{j}\right) \delta\left(s_{i}, s_{j}\right)
$$

because $1+s_{i} s_{j}=2 \delta\left(s_{i}, s_{j}\right)$. This is the $2 q$-state Potts model. For the square lattice the critical coupling is $J_{C}=\frac{1}{2} \ln (1+\sqrt{2 q})$ which corresponds to $w_{C}=\sqrt{2 q}$ or $p_{C}=\sqrt{2 q} /(1+\sqrt{2 q})$. For the random-bond percolation $q=1$ and thus $p_{C}=2-\sqrt{2}$.

If $p=1$ or $w=\infty$ from Eq. (5) we find $J=\infty$ and $L=K$. Since $\delta\left(\sigma_{i}, \sigma_{j}\right)=1$ if $J=\infty$, the Hamiltonian reduces to (apart from an additive constant) the pure Ising model: $-H / k_{B} T=\sum K s_{i} s_{j}$. The critical coupling for the square lattice is $K_{C}=\frac{1}{2} \ln (1+\sqrt{2})$.

For the random-bond percolation $q=1$, i.e., the Potts spin takes one value. Consequently $\delta\left(\sigma_{i}, \sigma_{j}\right)=1$ and the Hamiltonian of Eq. (6) becomes the Ising Hamiltonian: $-H / k_{B} T=\sum\left(L s_{i} s_{j}+J\right)$. We find by using Eq. (5) the Ising critical line in the parameter $w, K$ plane:

$$
\exp \left(2 L_{C}\right)=(1+w) /\left(1+w e^{-2 K}\right),
$$

where $L_{C}$ is the critical coupling of the pure Ising model. In the parameter plane of bond-occupation probability $p=w /(1+w)$ and $r=1-e^{-2 K}$ this equation becomes

$$
p r=1-\exp \left(-2 L_{C}\right) \text {. }
$$

For the square lattice $\exp \left(2 L_{C}\right)=1+\sqrt{2}$, and thus Eq. (9) reduces to

$$
p r=2-\sqrt{2} \text {. }
$$

\section{SOLUTION AND PHASE DIAGRAM}

We study the model by using the Migdal-Kadanoff renormalization-group scheme. ${ }^{6}$ The main asset of this scheme, besides its simplicity, is the fact that it is exact ${ }^{8}$ for the diamond hierarchical lattice. ${ }^{9}$ Hence physical requirements such as the positivity of the heat capacity and the exact results discussed in Sec. II above are satisfied by this renormalization scheme. $j$ is

The Boltzmann weight for bond connecting sites $i$ and

$$
Z_{i j}=1+\delta\left(\sigma_{i}, \sigma_{j}\right) w \exp \left[K\left(s_{i} s_{j}-1\right)\right] .
$$

The renormalized Boltzmann weight for a $2 \mathrm{D}$ lattice is

$$
Z_{i j}^{\prime}=\left\{\operatorname{Tr}_{\sigma_{k}} \operatorname{Tr}_{s_{k}} Z_{i k} Z_{k j}\right\}^{2}
$$

The following recursion equations are obtained by using Eqs. (11) and (12):

$w^{\prime}=\left[1+\frac{1}{2}\left(w^{2}+w^{2} e^{-4 K}\right) /\left(q+w+w e^{-2 K}\right)\right]^{2}-1$,

$w^{\prime} e^{-2 K^{\prime}}=\left[1+w^{2} e^{-2 K} /\left(q+w+w e^{-2 K}\right)\right]^{2}-1$.

The phase diagram in the parameter plane of $r=1-e^{-2 K}$ and $p=w /(1+w)$ at fixed $q$ is determined by following the recursion Eqs. (13) and (14). For all $q$ there are three phases: (i) the nonpercolating paramagnetic phase governed by the fixed point at $r=0(K=0$ or $T=\infty$ ) and $p=0$; (ii) the percolating paramagnetic phase governed by the fixed point at $r=0(K=0$ or $T=\infty)$ and $p=1$; (iii) the percolating ferromagnetic phase governed by the fixed point at $r=1(K=\infty$ or $T=0)$ and $p=1$. The ferromagnetic phase is always inside the percolating phase. In other words, there is no nonpercolating ferromagnetic phase. This result is consistent with our expectation that long-range order (ferromagnetism) requires an infinite percolating cluster.

For all $q$ values the percolating ferromagnetic phase is separated from the percolating paramagnetic phase by the Ising critical line governed by the fixed point at $p=1$ and $K=0.6094$ [exact value for square lattice coupling is $\left.\frac{1}{2} \ln (1+\sqrt{2})\right]$.

The percolating paramagnetic phase is separated from the nonpercolating paramagnetic phase by the percolation critical line which belongs to the universality class of the $q$-state Potts critical point. The fixed point governing this line is located at $r=0(K=0$ or $T=\infty)$.

Within the Migdal-Kadanoff renormalization group we find (see Fig. 1) that for $q \leq 8$ the percolation and Ising critical lines meet at a zero temperature $(r=1)$ point which belongs to the universality class of the $2 q$-state Potts model. The critical exponent corresponding to the flow along the $r=1$ axis is the thermal exponent of the

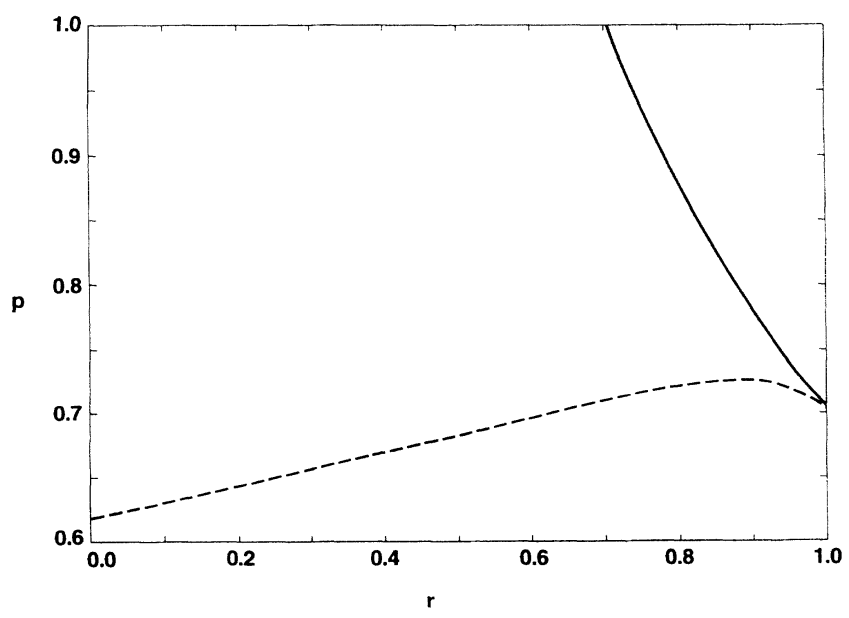

FIG. 1. Phase diagram for $q=1$ : solid line is Ising critical line; dotted line is percolation critical line. The two lines meet at $T=0$ at a point in the universality class of the $2 q$-state Potts model. 


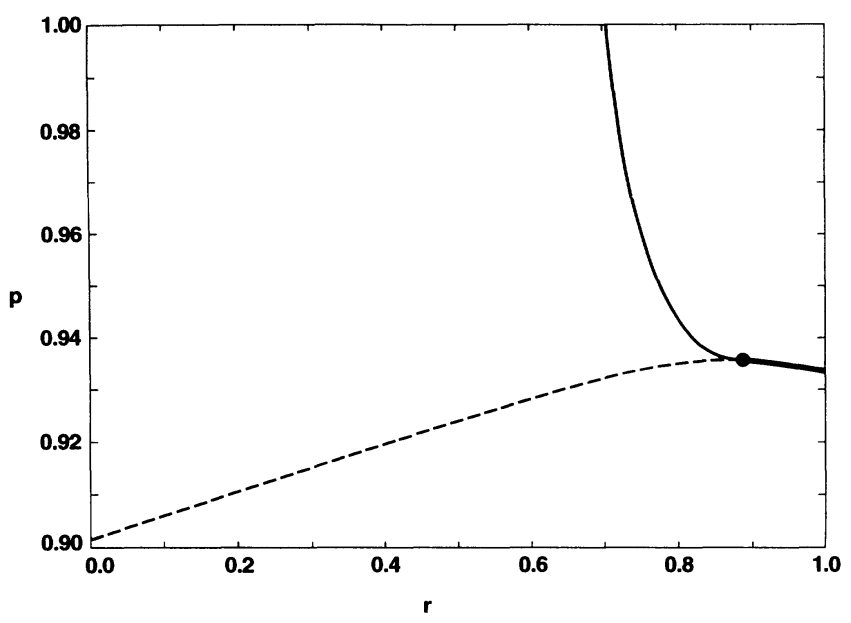

FIG. 2. Phase diagram for $q=20$ : solid line is Ising critical line; dotted line is percolation critical line; the double solid line is in the universality class of the $2 q$-state Potts model. The three lines meet at a multicritical point.

$2 q$-state Potts model. This is consistent with the exact result derived in Sec. II according to which at $T=0$ the model reduces to the $2 q$-state Potts model. For a small range of $p$ values we observe the following reentrance phenomenon. As $T$ is lowered ( $r$ increased) at fixed $p$ the following phases are encountered: the percolating paramagnetic, the nonpercolating paramagnetic, the percolating paramagnetic, and the percolating ferromagnertic phase.

For $q>8$ the percolation critical line (universality class of $q$-state Potts model) meets the Ising critical line at a novel multicritical point, as shown in Fig. 2. The critical exponents determined by linearizing the recursion equations are positive; i.e., this is an unstable fixed point. The critical line staring at this multicritical point and ending at the zero temperature $(r=1)$ critical fixed point is in the universality class of the $2 q$-state Potts model. This latter line separates the percolating ferromagnetic phase from the nonpercolating paramagnetic phase.

\section{SUMMARY AND CRITIQUE}

We presented a study of the phase diagram of the Ising model on percolation clusters. The percolation part of this problem is a generalization of the random-bond percolation which includes a fugacity $q$ controlling the number of clusters. By mapping the model on a Potts-Ising model and studying the latter we derived a number of exact results. In particular for the Ising model on random percolation clusters $(q=1)$ the Ising critical line is $p\left(1-e^{-2 K}\right)=1-\exp \left(-2 L_{C}\right)$, where $L_{C}$ is the pure Ising model critical coupling.

The phase diagram of the model was determined by using the Migdal-Kadanoff renormalization-group scheme. For small $q$ the Ising critical line and the percolation critical line intersect at a $2 q$-state Potts critical point located at $T=0$. For large $q$ the two lines meet at a novel multicritical point at nonzero temperature. In this case the percolating ferromagnetic phase can be accessed from the nonpercolating paramagnetic phase across a line in the universality class of the $2 q$-state Potts critical point.

The scheme used in this work gives continuous transitions for all $q$ while it is known ${ }^{5}$ that the 2D Potts model has a discontinuous transition for $q>4$. We do then expect that the multicritical point in Fig. 2 is replaced by a critical end point. ${ }^{10}$ Numerical simulations are needed to verify the predictions of this work.
*Present address: Department of Physics, Auburn University, Auburn, Alabama 36849-5311.

${ }^{1}$ D. W. Pohl and W. I. Goldburg, Phys. Rev. Lett. 48, 1111 (1982); J. V. Maher, W. I. Goldburg, D. W. Pohl, and M. Lanz, ibid. 53, 60 (1984); L. V. Entov, V. A. Levchenko, and V. P. Voronov, Int. J. Thermophys. 14, 221 (1993).

${ }^{2}$ M. Kaufman and D. Andelman, Phys. Rev. B 29, 4010 (1984).

${ }^{3}$ R. H. Swendsen and J.-S. Wang, Phys. Rev. Lett. 58, 86 (1986).

${ }^{4}$ C.-K. Hu, in Proceedings of the 1986 Summer School on Statistical Mechanics, edited by C.-K. Hu (Institute of Physics, Academia Sinica and Physical Society of the Republic of China, Taipei, 1987), p. 91; T. A. Larsson, J. Phys. A 19, 2383 (1986).
${ }^{5}$ F. Y. Wu, Rev. Mod. Phys. 54, 235 (1982).

${ }^{6}$ A. A. Migdal, Zh. Eksp. Teor. Fiz. 69, 1457 (1975) [Sov. Phys. JETP 42, 743 (1976)]; L. P. Kadanoff, Ann. Phys. (N.Y.) 100, 359 (1976).

${ }^{7}$ A. N. Berker and S. Ostlund, J. Phys. C 12, 4961 (1979).

${ }^{8}$ R. B. Griffiths and M. Kaufman, Phys. Rev. B 26, 5022 (1982); M. Kaufman and R. B. Griffiths, ibid. 30, 244 (1984).

${ }^{9}$ P. W. Kasteleyn and C. M. Fortuin, J. Phys. Soc. Jpn. 26, 11 (1969).

${ }^{10}$ M. Kaufman, R. B. Griffiths, J. M. Yeomans, and M. E. Fisher, Phys. Rev. B 23, 3448 (1981); M. E. Fisher and P. J. Upton, Phys. Rev. Lett. 65, 2402 (1990); R. R. Netz and A. N. Berker, Phys. Rev. B 47, 15019 (1993). 